\title{
Age-associated striatal dopaminergic denervation and falls in community-dwelling subjects
}

\author{
Nicolaas I. Bohnen, MD, PhD; ${ }^{1-2 *}$ Martijn L. T. M. Muller, PhD; ${ }^{2}$ Hiroto Kuwabara, MD, PhD; ${ }^{3}$ Rakié \\ Cham, PhD; ${ }^{4}$ Gregory M. Constantine, PhD; ${ }^{5}$ Stephanie A. Studenski, MD, MPH ${ }^{6-7}$ \\ ${ }^{1}$ Department of Veterans Affairs (VA) Ann Arbor Healthcare System, Geriatric Research, Education, and Clinical \\ Center (GRECC), Ann Arbor, MI; ${ }^{2}$ Functional Neuroimaging, Cognitive and Mobility Laboratory, Departments \\ of Radiology and Neurology, University of Michigan, Ann Arbor, MI; ${ }^{3}$ Department of Radiology, Johns Hopkins \\ University, Baltimore, MD; Departments of ${ }^{4}$ Bioengineering and ${ }^{5}$ Mathematics and Statistics, University of Pittsburgh, \\ Pittsburgh, PA; ${ }^{6}$ VA Pittsburgh Healthcare System, GRECC, Pittsburgh, PA; ${ }^{7}$ Department of Medicine, Division of \\ Geriatric Medicine, University of Pittsburgh Medical School, Pittsburgh, PA
}

\begin{abstract}
Older adults have a high prevalence of gait and balance disturbances and falls. Normal aging is associated with significant striatal dopaminergic denervation, which might be a previously unrecognized additional contributor to geriatric falls. This study investigated the relationship between the severity of age-associated striatal dopaminergic denervation (AASDD) and falls in community-dwelling subjects. Community-dwelling subjects who did not have a clinical diagnosis to explain falls $(n=77$ : 43 female, 34 male; mean age $61.4+/-16.4$; range 20-85) completed clinical assessment and brain dopamine transporter (DAT) $\left[{ }^{11}\right.$ C]beta-CFT (2-beta-carbomethoxy-3beta-(4-fluorophenyl) tropane) positron emission tomography imaging followed by 6 months of prospective fall monitoring using diaries. Results showed a significant inverse relationship between striatal DAT activity and age $(r=-0.82, p<0.001)$. A total of 26 subjects (33.8\%) reported at least one fall, with 5 subjects $(6.5 \%)$ reporting two or more falls. While no significant difference was noted in striatal DAT activity between nonfallers $(n=51)$ and fallers ( $n=26 ; f=0.02$, not significant), striatal DAT activity was modestly reduced in the small subgroup of recurrent fallers compared with the other subjects $(f=5.07, p<0.05)$. Findings indicate that AASDD does not explain isolated self-reported falls in community-dwelling subjects. However, it may be a contributing factor in the small subgroup of subjects with recurrent falls.
\end{abstract}

Key words: AASDD, age-associated striatal dopaminergic denervation, aging, basal ganglia, dopamine transporter, fall diary, falls, nigrostriatal, Parkinson, positron emission tomography, recurrent falls.

\section{INTRODUCTION}

Falls are a common problem in the elderly population [1-3]. In community-dwelling individuals aged 65 and older, approximately one-third fall every year and about half of these do so recurrently [1,4-5]. Falls are considered a multifactorial syndrome in which multiple contributing factors accumulate to disturb gait and balance [6]. Detectable gait abnormalities are present in 20 to 40 percent of

\footnotetext{
Abbreviations: 3-D = three-dimensional, AASDD = ageassociated striatal dopaminergic denervation, ANCOVA = analysis of covariance, BMI = body mass index, DAT = dopamine transporter, ICD-9 = International Classification of Diseases-9th Revision, MMSE = Mini-Mental State Examination, $\mathrm{MR}=$ magnetic resonance, $\mathrm{MRI}=\mathrm{MR}$ imaging, $\mathrm{PD}=$ Parkinson disease, $\mathrm{PET}=$ positron emission tomography, $\mathrm{SD}=$ standard deviation, SPGR = spoiled gradient recall, UPDRS = Unified Parkinson's Disease Rating Scale, VA = Department of Veterans Affairs, VOI = volume of interest.

* Address all correspondence to Nicolaas I. Bohnen, MD, PhD; Functional Neuroimaging, Cognitive and Mobility Laboratory, Departments of Radiology and Neurology, University of Michigan, 24 Frank Lloyd Wright Drive, Box 362, Ann Arbor, MI 48105-9755; 734-936-5388; fax: 734998-8403. Email: nbohnen@umich.edu

DOI:10.1682/JRRD.2009.03.0030
} 
persons over 65 and increase to 50 percent in those older than 85 [7]. Known contributors to falls include sensory disorders, cognitive and movement disorders, arrhythmias, orthostatic hypotension, and musculoskeletal problems [8]. While patients with Parkinson disease (PD) are known to have a very high prevalence of falls, the contribution to falls risk of non-PD, age-related decrease in dopaminergic innervation is not known.

Although not as severe as in PD, age-related dopaminergic changes in the striatum are significant [9] and are in the range of about 5 to 7 percent per decade as reported by in vivo positron emission tomography (PET) imaging [1011]. It is unclear whether these age-associated striatal losses are solely the result of frank neuronal loss of dopaminergic cells in the substantia nigra or involve other mechanisms such as axonal or synaptic dysfunction [12-14].

Volkow et al. were the first to show that age-related decreases in brain dopamine activity in nonparkinsonian elderly are associated with a decline in motor function [15-16]. The fact that this association is observed in nondisabled subjects indicates that while no typical PD symptoms are present, the decline in brain dopamine activity may lead to a subclinical threshold decline in an individual's balance and gait performance [17-18]. As falls are common and disabling, even in relatively early stage PD [19], the question then arises whether age-associated striatal dopaminergic denervation (AASDD) may be a possible mechanism contributing to geriatric falls. The present study investigated the relationship between severity of AASDD and prospectively ascertained falls in community-dwelling subjects. We hypothesized that fallers, especially those with recurrent falls, would have lower striatal dopamine transporter (DAT) activity than nonfallers.

\section{SUBJECTS AND METHODS}

\section{Subjects and Clinical Testing}

A total of 113 subjects ( 63 female and 50 male) between the ages of 20 and 85 were enrolled in this study, and 77 subjects completed the full clinical fall assessment and PET imaging test battery (see next section for details). Written informed consent, approved by the Institutional Review Boards of the Department of Veterans Affairs (VA) Pittsburgh Healthcare System and the University of Pittsburgh, was obtained prior to subject participation. All subjects underwent a medical and neurological examination. Clinical assessment included a neurological examination, including visual acuity measurement, Unified Parkinson's Disease Rating Scale (UPDRS) parkinsonian motor examination [20], measurement of orthostatic vital signs, weight, and height. The Berg Balance Scale was used to assess clinical balance function [21]. Lower-limb large-fiber sensory function was assessed by using a $128 \mathrm{~Hz}$ tuning fork to measure the duration of perception of vibratory sensations at each ankle. Subjects with clinical evidence of defined impairments that affect balance or gait were excluded. These impairments included orthostatic hypotension (defined as drop in systolic blood pressure of $>20 \mathrm{~mm} \mathrm{Hg}$ upon standing), impaired vision ( $<20 / 40$ corrected in both eyes), vertiginous disorder (defined by clinical symptoms of linear or rotational illusion of motion), myelopathy (defined by neurological examination findings of hyperreflexia, weakness and/or sensory level), myopathy (defined by presence of muscle weakness and prior laboratory evidence of a myopathic process), radicular (defined by examination findings of decreased or absent deep tendon reflexes with segmental weakness or dermatomal sensory loss), or cerebellar (clinical examination findings of limb or gait ataxia) syndromes. The UPDRS was used to rate the presence of nonspecific parkinsonian-like motor impairments often associated with normal aging [20,22]. Subjects with DAT PET evidence of PD, i.e., (asymmetric) gradient of putaminal more than caudate nucleus dopaminergic denervation [11], were excluded from this study. This DAT PET PD pattern is distinctly different from the age-associated pattern of symmetric and evenly distributed mild loss of dopaminergic activity in the putamen and caudate nucleus [11].

Additional exclusion criteria included a history of joint prosthetic surgery; subjects taking central nervous system suppressant medications, such as benzodiazepines, barbiturates, or skeletal muscle relaxants; or presence of dementia (Mini-Mental State Examination [MMSE] <24 [23]). Subjects with evidence of large-vessel stroke, tumor, focal intracranial lesion, or significant leukoaraiosis, i.e., stage $>3$ on the Brandt-Zawadzki et al. scale [24], on brain magnetic resonance (MR) were excluded from the study.

Falls were assessed prospectively using monthly diaries with telephone support $[8,25]$. We defined a fall according to International Classification of Diseases-9th Revision (ICD-9) criteria (ICD-9: E880A-E889A) as an unexpected event in which a person falls to the ground from an upper level or the same level. Fallers were classified as recurrent (two or more falls within 6 months) or nonrecurrent. 
Of the original 113 participants, 5 were excluded: 2 subjects were found to have PD on neurological examination and/or DAT PET imaging and 3 had abnormal MR findings. The mean age ( \pm standard deviation [SD]) of the remaining 108 subjects was $63.2 \pm 15.4$ years (range 20-85 years; 60 female and 48 male). Of the 108 subjects, 89 completed the 6-month prospective fall diary study (mean age 62.5 \pm 16.1 years, range 20-85 years; 51 female and 38 male). From this sample of 89 subjects, 77 subjects also completed striatal DAT PET imaging (mean age 61.4 \pm 16.4 years, 20-85 years; 43 female and 34 male) and were included in the primary analysis in this study. Compared with the 77 subjects included in this analysis, the 31 who were not included did not differ significantly in sex or fall status, but excluded subjects tended to be older (mean age $67.9 \pm 11.3$ years).

\section{Dopamine Transporter Positron Emission Tomography and Magnetic Resonance Imaging}

$\left[{ }^{11} \mathrm{C}\right] \beta$-CFT (2- $\beta$-carbomethoxy-3 $\beta$-(4-fluorophenyl) tropane) or $\left[{ }^{11} \mathrm{C}\right]$-WIN 35,428 is a specific radioligand for DAT. $\left[{ }^{11} \mathrm{C}\right] \beta$-CFT was prepared using a previously described method [26-27]. Dynamic PET scanning was performed for 90 minutes following a bolus intravenous injection of $370 \mathrm{MBq}$ of $\left[{ }^{11} \mathrm{C}\right] \beta$-CFT. Sequential emission scans were obtained in three-dimensional (3-D) imaging mode using an ECAT HR+ tomograph (CTI PET Systems; Knoxville, Tennessee), which acquires 63 transaxial slices (axial field of view: $15.2 \mathrm{~cm}$; slice thickness: $2.4 \mathrm{~mm}$ with an inplane resolution of $4.1 \mathrm{~mm}$ ). A thermoplastic mask was made for each subject to minimize head movement. The scanner gantry was equipped with a Neuroinsert (CTI PET Systems) to reduce the contribution of scattered photon events [28]. PET emission data were corrected for attenuation, scatter, and radioactive decay.

A volumetric spoiled gradient recall (SPGR) MR image was collected for each subject using a Signa 1.5 Tesla scanner (GE Medical Systems; Milwaukee, Wisconsin) with a standard head coil. The coronal SPGR sequence (echo time $=5$, repetition time $=25$, flip angle $=40^{\circ}$, number of excitations $=1$, slice thickness $=1.5 \mathrm{~mm}$, image matrix $=256 \times 192$, field of view $=24 \mathrm{~cm}$ ) was acquired to maximize contrast among gray matter, white matter, and cerebral spinal fluid and provide high-resolution delineation of cortical and subcortical structures. Volumes of interest (VOIs) were defined manually on the SPGR MR imaging (MRI) for striatum (caudate nucleus and putamen) with left and right hemispheres combined by manu- ally tracing the outlines of the structures in 3-D using software developed in-house (VOILand). VOIs were transferred from MRI space to PET space using MRI-to-PET coregistration parameters that were obtained with the mutual information theory [29] as implemented in the intermodality coregistration module of the SPM2 image analysis software package (Wellcome Trust Centre for Neuroimaging; London, United Kingdom) [30]. VOIs were applied on successive dynamic PET frames to generate time radioactivity curves of the regions.

Regional cerebral $\left[{ }^{11} \mathrm{C}\right] \beta-\mathrm{CFT}$ binding was determined by graphical analysis of the bolus-plus-infusion transformation plot of bolus-only PET experiments [31]. Because of the relatively slow dissociation of $\left[{ }^{11} \mathrm{C}\right] \beta$ $\mathrm{CFT}$, the method that yielded the net uptake constant $\left(\mathrm{K}_{i}\right)$ of $\left[{ }^{11} \mathrm{C}\right] \beta$-CFT was shown to be more robust in detecting early changes in DAT in experimental PD than methods that assumed dissociation of the ligand and yielded binding potential [31]. Results of associations between striatal DAT and specific aspects of gait and balance from this series have been previously reported [17-18].

\section{Data Analysis}

Analysis of covariance (ANCOVA) was performed to compare fallers with nonfallers while controlling for the effects of age. Analyses were performed with SAS (version 9.1, SAS Institute, Inc; Cary, North Carolina).

\section{RESULTS}

\section{Study Demographics, Clinical Variables, and Prospective Falls}

Of the 77 subjects who completed the DAT PET study, 26 were fallers (including 4 with 2 falls and 1 with 4 falls) and 51 were nonfallers. No significant difference was noted in mean age between the 26 fallers $(64.7 \pm 16.8)$ and 51 nonfallers (59.8 $\pm 16.2, t=1.25$, not significant). No significant difference was noted in sex between fallers (57.7\% females) and nonfallers ( $54.9 \%$ females; $\chi^{2}=0.05$, $p>0.05)$.

The Table lists information on clinical variables in the different subgroups of control subjects and single-time and recurrent fallers. Although no significant age difference was noted between the groups, the recurrent fallers tended to be older (72.8 \pm 16.9 ; Table). No significant differences were found between the subgroups for the clinical variables of body mass index (BMI), lower-limb 
Table.

Demographic and clinical information (mean \pm standard deviation) unless otherwise noted of single-time and recurrent fallers and nonfallers who completed 6-month prospective fall diary and positron emission tomography imaging study. For significant overall group effects, Duncan's Multiple Range post hoc testing was used to compare differences between subgroups.

\begin{tabular}{|c|c|c|c|c|}
\hline Variable & $\begin{array}{l}\text { Nonfallers } \\
(n=51)\end{array}$ & $\begin{array}{l}\text { Single-Time Fallers } \\
\qquad(n=21)\end{array}$ & $\begin{array}{l}\text { Recurrent Fallers } \\
\qquad(n=5)\end{array}$ & Group Effect \\
\hline $\operatorname{Sex}(n: \mathrm{F} / \mathrm{M})$ & $28 / 23$ & $11 / 10$ & $4 / 1$ & $\chi^{2}=1.30^{*}$ \\
\hline Body Mass Index $\left(\mathrm{kg} / \mathrm{m}^{2}\right)$ & $27.2 \pm 5.2$ & $26.9 \pm 4.9$ & $25.3 \pm 2.7$ & $f=0.32^{*}$ \\
\hline MMSE & $29.5 \pm 0.8$ & $29.5 \pm 0.9$ & $29.8 \pm 0.5$ & $f=0.32^{*}$ \\
\hline Motor UPDRS & $3.3 \pm 3.8$ & $3.5 \pm 4.6$ & $4.2 \pm 4.7$ & $f=0.13^{*}$ \\
\hline Ankle Vibration (s) & $10.4 \pm 4.5$ & $10.4 \pm 5.4$ & $13.5 \pm 2.6$ & $f=1.03^{*}$ \\
\hline
\end{tabular}

sensory function, UPDRS motor scores, and MMSE (Table). Recurrent fallers had significantly lower scores on the Berg Balance Scale than nonfallers (Table). However, scores on the Berg Balance Scale in the single-time fallers trended to be in the intermediate range between the control and recurrent fallers groups.

\section{Striatal Dopamine Transporter Activity, Aging, and Falls}

A significant inverse relationship was found between age and striatal DAT activity $(r=-0.82, p<0.001)$. Correlation coefficients between age and striatal DAT activity were identical for females and males, $r=-0.82, p<0.001$. The Figure is a scatter plot for striatal DAT activity and age by fall status.

ANCOVA was used to compare striatal DAT activity between fallers (combined single-time and recurrent fallers) and nonfallers while adjusting for the effects of age. No significant group differences were found in striatal DAT activity between nonfallers and fallers: age-adjusted striatal DAT activity for nonfallers was $0.0185 \pm 0.0029$ and for fallers was $0.0185 \pm 0.0033(f=0.01, p>0.05)$.

A subgroup analysis was performed to compare the subgroup of recurrent fallers with the other subjects (singletime fallers and nonfallers). ANCOVA analysis demonstrated that striatal DAT activity was modestly reduced $(f=5.07, p=0.03)$ in recurrent fallers $(0.0168 \pm 0.0035)$ compared with other subjects $(0.0186 \pm 0.0029)$.

\section{Post Hoc Analysis: Clinical Correlates of AASDD in Subjects 65 and Older}

As AASDD is expected to have more clinical significance in the elderly, we performed a post hoc analysis to evaluate clinical correlates of striatal dopaminergic denervation in the subgroup of elderly aged 65 and over $(n=$ 35). Age-adjusted ANCOVA analysis limited to the elderly demonstrated a significant group effect $(f=5.46, p=0.03)$ of reduced striatal DAT activity in the recurrent elderly fallers $(0.0141 \pm 0.0020)$ compared with other subjects $(0.0166 \pm 0.0019)$. Significant correlations were also found between lower striatal DAT and higher scores on the UPDRS motor ( $r=-0.39, p=0.02)$, but no significant correlations were found with MMSE, Berg Balance Scale, BMI, or ankle vibration sense scores.

Subjects were also classified as AASDD if their striatal DAT binding was $>3$ SD below the mean of that in the younger age group (20 to 49 years). Following these criteria, 9 elderly had AASDD and 26 had striatal DAT levels higher than this cutoff (NO-AASDD). As expected, risk of falling was higher in the AASDD than the NO-AASDD subjects $\left(\chi^{2}=6.4, p=0.04\right)$ and AASDD subjects had a trend toward higher UPDRS motor scores $(7.9 \pm 6.2)$ than NO-AASDD subjects ( $4.5 \pm$ $4.0, t=1.86, p=0.08)$. No significant differences in age, Berg Balance Scale, MMSE, BMI, or ankle vibration sense scores were noted between the AASDD and NOAASDD groups. 


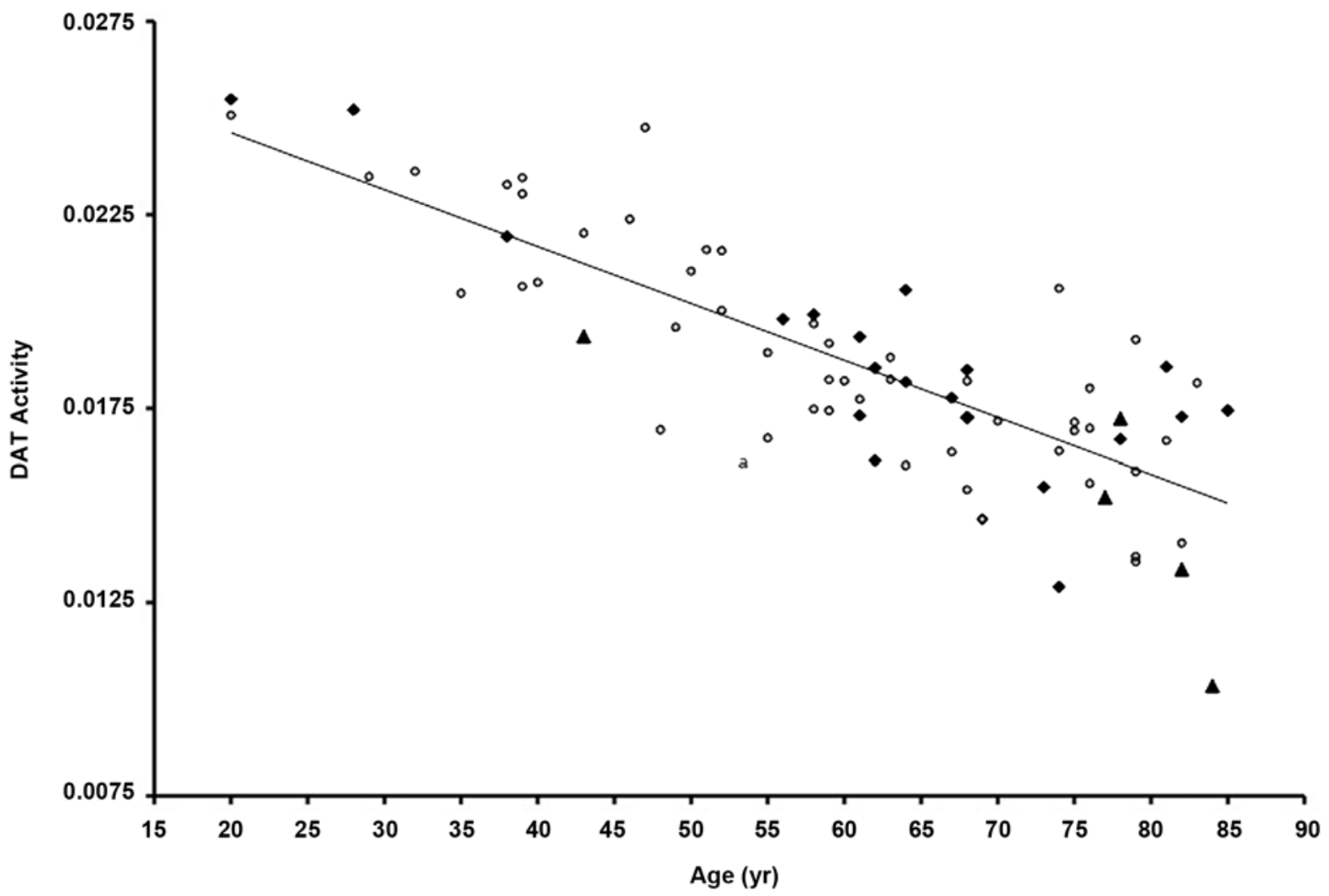

Figure.

Striatal dopamine transporter (DAT) activity versus age and fall status: Nonfaller $=$ open circles, single-time faller $=$ black rhombus, recurrent faller = black triangle. Plot shows significant age-associated nigrostriatal dopaminergic denervation. Linear regression line is presented. No overall significant difference was noted in nigrostriatal dopaminergic activity between fallers and nonfallers.

\section{DISCUSSION}

Of the age-related neurochemical changes in the brain, those involving the dopamine system appear to be of paramount importance [9]. Although an average person may lose about 33 percent of striatal dopaminergic innervation between the ages of 25 and 75 [10], this loss is not as severe as in PD, where losses often exceed 50 to 80 percent [32]. We should note, however, that normal aging is associated with substantial interindividual variability in dopaminergic losses, with some elderly having more pronounced AASDD while others have relatively preserved activity. However, despite a large number of studies documenting significantly decreased dopamine activity with normal aging [10-11], very little is known about its functional significance. We previously reported findings of reduced striatal dopaminergic activity with normal aging from subjects in this series and found that, in normal aging, the central ability to inhibit balance-destabilizing vision-related pos- tural control processes depends at least partially on striatal dopaminergic pathways. In contrast, striatal dopaminergic denervation does not appear to impair the ability to disengage destabilizing proprioceptive inputs and trigger the vestibular control system during challenging sensory perturbations [17]. With respect to gait, we found that gait cadence was significantly slower than age-based predictions in adults with lower striatal DAT activity [18]. This result translated into slower speed and longer single- and double-support durations [18]. Despite this specific balanceand gait-related dopaminergic evidence, our present findings indicate that AASDD is not a significant factor to explain self-reported isolated falls in community-dwelling subjects. One potential explanation for this apparent difference in results is the multidimensional nature of the causes of falls. Indeed, it is well known that community-dwelling older adults can fall for many reasons and thus isolated "falls" may lack specificity to be correlated with AASDD. Our prior gait and balance studies have considered very 
specific aspects of the postural control system and thus may have been more sensitive to striatal dopaminergic denervations. Additionally, it is interesting to note that our preliminary results from a subgroup analysis in this current study suggest that AASDD may be a contributing factor in the small subgroup of subjects with recurrent falls. This finding may also suggest that the relationship between altered biomechanics and falls may be nonstochastic in nature; i.e., effects are characterized by a threshold dose below which they do not occur. Given the multifactorial nature of geriatric falls, it is also plausible that different risk factors, when present together, may have a synergistic detrimental effect on fall risk that is disproportionately larger than when a single risk factor is studied in isolation.

De Lau et al. studied subjective complaints that may precede the diagnosis of PD and found that subjects who reported imbalance at baseline had a significantly increased risk of PD at 2-year follow-up, with a hazard ratio of 3.47 [33]. Perceived imbalance may be a very early symptom that progresses to overt postural instability and an increased risk of falling later in the course of the disease. Our data may not necessarily indicate that the group of recurrent fallers is at risk of developing PD in the (very) near future, because DAT PET imaging in our study did not demonstrate evidence of the typical PD striatal topographic pattern since this represented an exclusion criterion. All subjects in this study, including those with mildly increased motor ratings on the UPDRS examination demonstrated evidence of diffuse striatal DAT loss with aging, i.e., a pattern of bilateral and symmetric striatal loss without evidence of predominant or asymmetric putaminal denervation that would indicate PD. Furthermore, the modest reductions of striatal DAT binding in the recurrent fallers are above typical denervation levels seen in PD [11]. The fact that this association is observed in otherwise nondisabled subjects indicates that, while no typical symptoms of PD are present, the decrease in age-associated brain dopamine activity may lead to a decline in the individual's balance and gait performance. This effectively would indicate that a minimally symptomatic hypodopaminergic state of aging exists that potentially could benefit from dopaminergic therapy yet is distinct from idiopathic PD. We should also note that PD is now being recognized as a multisystem disorder in which nigrostriatal dopaminergic denervation is only one of many neuronal degenerations present in the peripheral and central nervous systems [34-35].

Results of our post hoc analysis of subjects 65 years and older demonstrated inverse correlations between scores on the motor UPDRS and striatal DAT activity but not with the Berg Balance Scale. A trend toward lower scores was also noted on the Berg Balance Scale in the group of the single-time fallers who did not differ in striatal DAT binding from nonfallers. Therefore, the mechanism underlying increased fall risk that may be related to age-associated decreases in striatal DAT binding does not appear to intrinsically overlap with the fall risk mechanism underlying abnormal performance on the Berg Balance Scale. We should note that the UPDRS specifically assesses motor impairments present in PD while the Berg Balance Scale represents a more global assessment of balance control.

A limitation of this study was the small number of recurrent fallers within our study sample. Another limitation of the study was that the duration of the diary study was only 6 months. Therefore, categorization of groups into fallers and nonfallers remains arbitrary given the limited study observation period. Furthermore, members of the single-time fallers group may have fallen for many reasons related to chance or extrinsic or accidental circumstances. In this context, recurrent fallers may represent a more reliable assessment of intrinsic fall risk in the elderly. This small subgroup also represents a disproportionately larger burden on healthcare costs [36].

\section{CONCLUSIONS}

We conclude that AASDD is not a significant factor to explain self-reported isolated falls in otherwise nondisabled subjects. However, it may be a contributing factor in the small subgroup of subjects with recurrent falls.

\section{ACKNOWLEDGMENTS}

\author{
Author Contributions: \\ Study concept and design: N. I. Bohnen, S. A. Studenski. \\ Acquisition of data: N. I. Bohnen, S. A. Studenski. \\ Analysis and interpretation of data: N. I. Bohnen, M. L. T. M. Muller, \\ H. Kuwabara. \\ Drafting of manuscript: N. I. Bohnen, M. L. T. M. Muller. \\ Critical revision of manuscript for important intellectual content: \\ H. Kuwabara, G. M. Constantine, R. Cham, S. A. Studenski. \\ Statistical analysis: N. I. Bohnen, G. M. Constantine, \\ M. L. T. M. Muller. \\ Obtained funding: N. I. Bohnen, S. A. Studenski. \\ Administrative, technical, or material support: R. Cham. \\ Study supervision: N. I. Bohnen, S. A. Studenski.
}


Financial Disclosures: The authors have declared that no competing interests exist.

Funding/Support: This material was based on work supported by VA and the National Institutes of Health National Institute on Aging (grants AG023641 and AG024827).

Additional Contributions: We would like to thank Susan Marcanio and Christine Minderovic for assistance with the study.

Participant Follow-Up: The authors do not plan to notify the participants of the publication of this article because of a lack of contact information.

\section{REFERENCES}

1. Tinetti ME, Speechley M, Ginter SF. Risk factors for falls among elderly persons living in the community. $\mathrm{N}$ Engl J Med. 1988;319(26):1701-7. [PMID: 3205267]

2. Studenski S, Rigler SK. Clinical overview of instability in the elderly. Clin Geriatr Med. 1996;12(4):679-88.

[PMID: 8890110]

3. Kannus P, Parkkari J, Koskinen S, Niemi S, Palvanen M, Järvinen M, Vuori I. Fall-induced injuries and deaths among older adults. JAMA. 1999;281(20):1895-99.

[PMID: 10349892]

DOI:10.1001/jama.281.20.1895

4. Tinetti ME, Speechley M. Prevention of falls among the elderly. N Eng J Med. 1989;320(16):1055-59.

[PMID: 2648154$]$

5. King MB, Tinetti ME. Falls in community-dwelling older persons. J Am Geriatr Soc. 1995;43(10):1146-54. [PMID: 7560708]

6. Duncan PW, Chandler J, Studenski S, Hughes M, Prescott B. How do physiological components of balance affect mobility in elderly men? Arch Phys Med Rehabil. 1993; 74(12):1343-49. [PMID: 8259903] DOI:10.1016/0003-9993(93)90090-W

7. Trueblood PR, Rubenstein LZ. Assessment of instability and gait in elderly persons. Compr Ther. 1991;17(8):20-29. [PMID: 1742974]

8. Tinetti ME. Clinical practice. Preventing falls in elderly persons. N Engl J Med. 2003;348(1):42-49. [PMID: 12510042] DOI:10.1056/NEJMcp020719

9. Joseph JA, Roth GS, Strong R. The striatum, a microcosm for the examination of age-related alterations in the CNS: A selected review. Rev Biol Res Aging. 1990;4:181-99.

10. Volkow ND, Ding YS, Fowler JS, Wang GJ, Logan J, Gatley SJ, Hitzemann R, Smith G, Fields SD, Gur R. Dopamine transporters decrease with age. J Nucl Med. 1996;37(4): 554-59. [PMID: 8691238]

11. Bohnen NI, Albin RL, Koeppe RA, Wernette KA, Kilbourn MR, Minoshima S, Frey KA. Positron emission tomography of monoaminergic vesicular binding in aging and Par- kinson disease. J Cereb Blood Flow Metab. 2006;26(9): 1198-1212. [PMID: 16421508]

12. Gibb WR, Lees AJ. Anatomy, pigmentation, ventral and dorsal subpopulations of the substantia nigra, and differential cell death in Parkinson's disease. J Neurol Neurosurg Psychiatry. 1991;54(5):388-96. [PMID: 1865199] DOI:10.1136/jnnp.54.5.388

13. Halliday GM, McRitchie DA, Cartwright H, Pamphlett R, Hely MA, Morris JG. Midbrain neuropathology in idiopathic Parkinson's disease and diffuse Lewy body disease. J Clin Neurosci. 1996;3(1):52-60. [PMID: 18644264] DOI:10.1016/S0967-5868(96)90083-1

14. Colebrooke RE, Humby T, Lynch PJ, McGowan DP, Xia J, Emson PC. Age-related decline in striatal dopamine content and motor performance occurs in the absence of nigral cell loss in a genetic mouse model of Parkinson's disease. Eur J Neurosci. 2006;24(9):2622-30. [PMID: 17100850] DOI:10.1111/j.1460-9568.2006.05143.X

15. Volkow ND, Gur RC, Wang GJ, Fowler JS, Moberg PJ, Ding YS, Hitzemann R, Smith G, Logan J. Association between decline in brain dopamine activity with age and cognitive and motor impairment in healthy individuals. Am J Psychiatry. 1998;155(3):344-49. [PMID: 9501743]

16. Volkow ND, Logan J, Fowler JS, Wang GJ, Gur RC, Wong C, Felder C, Gatley SJ, Ding YS, Hitzemann R, Pappas N. Association between age-related decline in brain dopamine activity and impairment in frontal and cingulate metabolism. Am J Psychiatry. 2000;157(1):75-80. [PMID: 10618016]

17. Cham R, Perera S, Studenski SA, Bohnen NI. Striatal dopamine denervation and sensory integration for balance in middle-aged and older adults. Gait Posture. 2007; 26(4):516-25. [PMID: 17196819] DOI:10.1016/j.gaitpost.2006.11.204

18. Cham R, Studenski SA, Perera S, Bohnen NI. Striatal dopaminergic denervation and gait in healthy adults. Exp Brain Res. 2008;185(3):391-98. [PMID: 17973106] DOI:10.1007/s00221-007-1161-3

19. Bloem BR, Grimbergen YA, Cramer M, Willemsen M, Zwinderman AH. Prospective assessment of falls in Parkinson's disease. J Neurol. 2001;248(11):950-58. [PMID: 11757958] DOI:10.1007/s004150170047

20. Fahn S, Elton RL; UPDRS Development Committee. Unified Parkinson's Disease Rating Scale. In: Fahn S, Marsden CD, Calne D, Holstein M, editors. Recent developments in Parkinson's disease. Florham Park (NJ): Macmillan Healthcare Information; 1987. p. 153-63.

21. Berg K, Wood-Dauphinee S, Williams JI. The Balance Scale: Reliability assessment with elderly residents and patients with an acute stroke. Scand J Rehabil Med. 1995; 27(1):27-36. [PMID: 7792547] 
22. Fleischman DA, Wilson RS, Schneider JA, Bienias JL, Bennett DA. Parkinsonian signs and functional disability in old age. Exp Aging Res. 2007;33(1):59-76.

[PMID: 17132564$]$ DOI:10.1080/03610730601006370

23. Folstein MF, Folstein SE, McHugh PR. "Mini-mental state”: A practical method for grading the cognitive state of patients for the clinician. J Psychiatr Res. 1975;12(3):189-98. [PMID: 1202204$]$ DOI:10.1016/0022-3956(75)90026-6

24. Brant-Zawadzki M, Fein G, Van Dyke C, Kiernan R, Davenport L, De Groot J. MR imaging of the aging brain: Patchy white-matter lesions and dementia. AJNR Am J Neuroradiol. 1985;6(5):675-82. [PMID: 3933292]

25. Studenski S, Duncan PW, Chandler J, Samsa G, Prescott B, Hogue C, Bearon LB. Predicting falls: The role of mobility and nonphysical factors. J Am Geriatr Soc. 1994;42(3): 297-302. [PMID: 8120315]

26. Någren K, Müller L, Halldin C, Swahn CG, Lehikoinen P. Improved synthesis of some commonly used PET radioligands by the use of [11C]methyl triflate. Nucl Med Biol. 1995;22(2):235-39. [PMID: 7767319] DOI:10.1016/0969-8051(94)00083-V

27. Någren K, Halldin C, Müller L, Swahn CG, Lehikoinen P. Comparison of [11C]methyl triflate and [11C]methyl iodide in the synthesis of PET radioligands such as [11C]beta-CIT and [11C]beta-CFT. Nucl Med Biol. 1995; 22(8):965-79. [PMID: 8998473] DOI:10.1016/0969-8051(95)02018-7

28. Weinhard K. Applications of 3D PET. In: Bendriem B, Townsend DW, editors. The theory and practice of 3D PET. Boston (MA): Kluwer Academic; 1998. p. 133-67.

29. Maes F, Collignon A, Vandermeulen D, Marchal G, Suetens P. Multimodality image registration by maximization of mutual information. IEEE Trans Med Imaging. 1997;16(2): 187-98. [PMID: 9101328] DOI:10.1109/42.563664
30. Ashburner J, Friston KJ. Rigid body registration. In: Frackowiak RS, Friston KL, Frith CD, Dolan RJ, Price C, Zeki S, editors. Human brain function. 2nd ed. San Diego (CA): Academic Press; 2004. p. 1-24.

31. Chen MK, Kuwabara H, Zhou Y, Adams RJ, Brasi JR, McGlothan JL, Verina T, Burton NC, Alexander M, Kumar A, Wong DF, Guilarte TR. VMAT2 and dopamine neuron loss in a primate model of Parkinson's disease. J Neurochem. 2008;105(1):78-90. [PMID: 17988241] DOI:10.1111/j.1471-4159.2007.05108.x

32. Bernheimer H, Birkmayer W, Hornykiewicz O, Jellinger K, Seitelberger F. Brain dopamine and the syndromes of Parkinson and Huntington. Clinical, morphological and neurochemical correlations. J Neurol Sci. 1973;20(4):415-55.

[PMID: 4272516] DOI:10.1016/0022-510X(73)90175-5

33. De Lau LM, Koudstaal PJ, Hofman A, Breteler MM. Subjective complaints precede Parkinson disease: The Rotterdam study. Arch Neurol. 2006;63(3):362-65.

[PMID: 16401729]

DOI:10.1001/archneur.63.3.noc50312

34. Braak H, Ghebremedhin E, Rüb U, Bratzke H, Del Tredici K. Stages in the development of Parkinson's diseaserelated pathology. Cell Tissue Res. 2004;318(1):121-34. [PMID: 15338272] DOI:10.1007/s00441-004-0956-9

35. Langston JW. The Parkinson's complex: Parkinsonism is just the tip of the iceberg. Ann Neurol. 2006;59(4):591-96. [PMID: 16566021] DOI:10.1002/ana.20834

36. Jansson B, Stenbacka M, Leifman A, Romelsjö A. A small fraction of patients with repetitive injuries account for a large portion of medical costs. Eur J Public Health. 2004; 14:161-67. [PMID: 15230502] DOI

Submitted for publication March 18, 2009. Accepted in revised form June 11, 2009. 